\title{
A Feature Comparative Study of Real-Time Traffic Simulation over OBS Network
}

\author{
Mohammed. A. Al-Shargabi \\ Faculty of Computer Science \& \\ Information Systems, \\ Universiti Teknologi Malaysia.
}

\author{
Abdul Samad Ismail \\ Faculty of Computer Science \& \\ Information Systems \\ Universiti Teknologi Malaysia.
}

\author{
Sevia M.Idrus \\ Faculty of Electrical Engineering \\ Photonic Technology Centre. \\ Universiti Teknologi Malaysia.
}

\begin{abstract}
Network simulator is a significant tool for researchers to simulate the actual network. The main advantage of the simulator is that it gives results much closer to the reality than the mathematical modeling. Furthermore, its cost is much lesser as compared to the real experimentation. The scale and size of the large networks (e.g. Internet) complicate both the mathematical modeling and the real experimentation. Therefore, researchers depend mainly on simulators to study, evaluate and design the performance of network. Optical Burst Switching (OBS) network is one of the most popular research areas in the optical Internet. However, it is just recently that some of these networks simulators start to provide the ability to simulate OBS network. In this paper, the concepts behind the some main OBS simulators are presented. Furthermore, a feature comparison is made among them in terms of the module-based structure, simulating methodology, compilation, and Graphic User Interface (GUI). The comparison of these simulators has been made based on the needs of simulating real time traffic over OBS network and not to define which network simulator is the best as there are too many different parameters variations and different possible network scenarios to adequately determine this in a single section. The comparison results show that NCTUns can provide much better functions and performance in the simulation of real time traffic in OBS network, because of its several unique features which are suitable for real time traffic over OBS.
\end{abstract}

\section{Keywords}

Network Simulators; OBS; Real time traffic.

\section{INTRODUCTION}

Recently, the Internet has become an active area of research due to the plenty of network services that it provides. Multimedia and real time traffic over the Internet attract more attention in the Internet research studies [1]. Optical Burst Switching (OBS) seems to be the next generation of the optical Internet infrastructure due to its attractive characteristics [2]. It combines the benefits of the circuit switching and packet switching method and it is designed for a better utilization of wavelengths, to minimize the latency (setup delay), and avoid the use of optical buffers. Therefore, resplendent studies in OBS network with real time traffic must be introduced.

Suitable simulator becomes one of the main parameters to succeed the research implementation. Thus, it is important to know the strengths and the weaknesses of the available simulation tools in order to allow users to select the most appropriate one for their needs. This paper studies three network simulators that involve OBS network simulation from different views.
This paper is organized with several sections. The next Section introduces network simulators. In section 3, Comparison among NCTUns, OBS-ns, and OPNET is introduced. Finally, section 4 concludes the paper.

\section{NETWORK SIMULATORS}

The network simulators (Weingartner et al., 2009, Soares et al., 2008) used to facilitate the testing and developing the new network protocols and architectures as significant tools for researchers to simulate the actual network. The main advantage of the simulation is that it gives results much closer to the reality than the mathematical modelling; and its cost is much lesser than the real experimentation.

Suitable simulator becomes one of the main parameters to succeed the research implementation. Thus, it is important to know the strengths and the weaknesses of the available simulation tools in order to allow researchers to select the most appropriate one for their needs. Accordingly, this paper introduces a comparative study of OBS network simulators for real-time traffic simulation to choose the most appropriate simulator to the case of this research in order to get more accurate results. The comparative study is introduced in the next section.

In this section, several network simulators that start to provide the ability to simulate the OBS network such as NCTUns (Quaglio et al., 2009), OBS-ns (Bo et al., 2001), OPNET (Xinjie, 1999), OBSsimulator, ADOBS simulator, and JAVOBS simulator have been introduced. Each one of them has its own characteristic and weaknesses. Consequently, more studies are needed to establish guidelines for researchers to identify the strengths and weaknesses of each simulator as to allow users to select the most appropriate one for their needs. The right choice will lead to more confidence in the results.

\subsection{National Chiao Tung University network simulator (NCTUns)}

The NCTUns (NCTU network simulator) is a network simulator developed by the Network and System Laboratory, CSIE, NCTU. Founded in 2002, SimReal Inc. is a virtual company promoting the use of the NCTUns network simulator and emulator.

The NCTUns simulates mainly the Open Systems Interconnection (OSI) layer-2 and layer-3 protocols such as routing and switching. In the OSI layer-1 (the physical layer), the NCTUns simulates the bit error rate, bandwidth and propagation delay. In all-optical networks, NCTUns provides two simulation environments: the first one is a traditional Optical circuit switching network, while the other one is the Optical burst switching network. Figure 1 shows the GUI for NCTUns. 


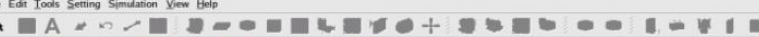
EEI

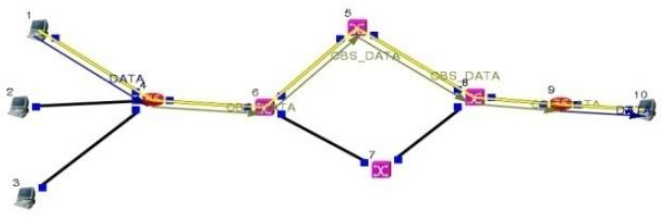

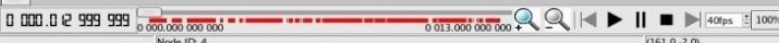

Fig. 1 The NCTUns Simulator GUI

\subsection{Optical Burst Switching-network simulator (OBS-ns)}

OBS-ns simulator is a network simulator based on Ns-2 (Network simulator version 2) and funded by the Optical Internet Research Centre (OIRC) and Samsung Advanced Institute of Technology (SAIT).

The Ns2 began as a variant of the real network simulator in 1989 , and it has substantially evolved over the past few years. In 1995, the Ns2 development was supported by DARPA through the VINT project at LBL, Xerox PARC, UCB, and USC/ISI. Currently, the Ns2 development is supported through DARPA with SAMAN and through NSF with CONSER; both in collaboration with other researchers, including the ACIRI. It has always included substantial contributions from other researchers. Figure 2 shows the GUI for OBS-ns.

\subsection{Optimized Network Engineering Tools (OPNET)}

Founded in 1986, the OPNET simulator is a network simulator developed by the OPNET Technologies, Inc. (NASDAQ: OPNT). OPNET provides network and application management software and hardware, as well as associated services. OPNET solutions address application performance management, network operations, and capacity management. An OPNET model for an optical burst switching network has been developed and verified. It can be used to model simple, single core node networks and multiple core node networks.

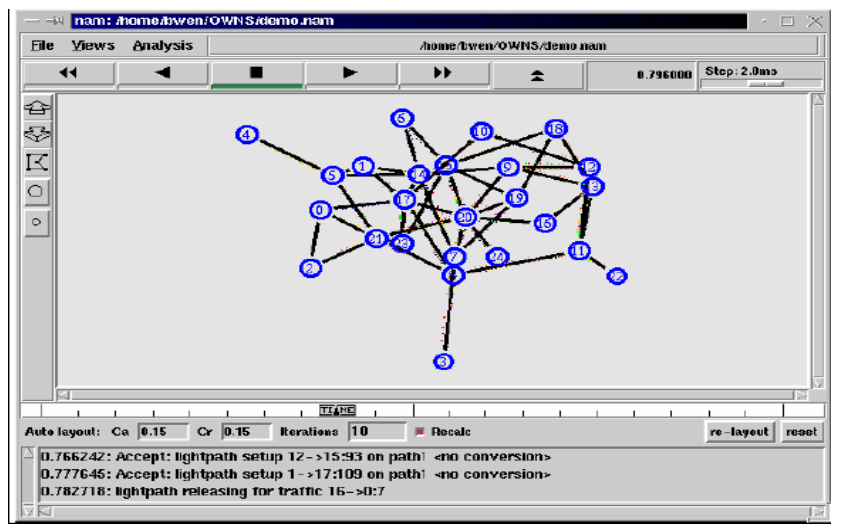

Fig. 2 The OBS-ns Simulator GUI

The model also takes into account different offset times in the JET protocol and the calculation of the offset time in the multiple core node networks, depending on the edge node.
Different burst assembly algorithms have been modeled, and they can be compared. Similarly, wavelength conversion and optical buffering (using fiber delay lines) can be modeled. Figure 3 shows the GUI for OPNET.

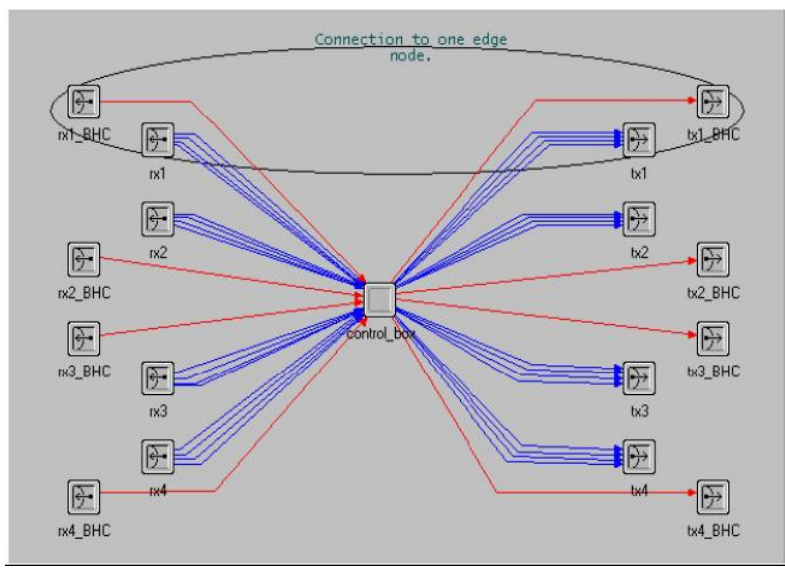

Fig. 3 The OPNET Simulator GUI

\subsection{OBSsimulator}

OBSsimulator is a network simulator developed by Teng and Rouskas from North Caroline State University, USA. In OBSsimulator, no graphic user interface is available. It uses a fixed file name (n1.net) with a particular semantics and syntax to run the simulator. Moreover, no animation view or documentation is provided .

\subsection{ADOBS Simulator}

The ADOBS simulator [4] is network simulator developed by M. Klinkowski from Technical University of Catalonia, USA Mainly, ADOBS simulates routing algorithms in OPS networks. However, a modified version has been provided to simulate OBS networks. ADOBS simulator is a product of a Ph.D's thesis. Thus, no enough support such as documentation or graphic user interface is available .

\subsection{JAVOBS simulator}

JAVOBS simulator is a network simulator developed by Oscar Pedrola from Technical University of Catalonia, USA also. In contrast to ADOBS Simulator, JAVOBS simulator is mainly developed to simulate OBS networks on the top of the JAVANCO framework.

\section{COMPARISON OF THE OBS NETWORK SIMULATORS}

In this section, a comparison among the mentioned above OBS network simulators is presented. This section does not aim to define which network simulator is the best as there are too many different parameters variations and different possible network scenarios to adequately determine this in a single section. Instead, this section demonstrates characteristics and weaknesses of each simulator in case of the needs of simulating real time traffic in the OBS network.

In any simulation model, providing the requirements of the simulated network environment is the most important issue that can affect the simulation results. In the case of real time traffic simulated in the OBS network, providing an enough amounts of traffic that could be created in case of the peak sending rate, or bursty sending rate is the main point that affects the result of the comparison of the OBS network simulators. 
In this section, a feature comparison among the simulators in terms of the module-based structure, simulating methodology, compilation, and Graphic User Interface (GUI), is introduced.

\subsection{Module Based Structure}

In the network simulators, the module-based structure is one of the main parameters that affect the results of the simulation process. In the module-based structure, supporting normal applications that generate the network traffic (traffic generators) and general network applications, such as utility programs to configure, monitor and gather statistical information about a simulated network, play a role in generating and analyzing the traffic.

One of the main issues in simulating optical traffic is the simulation of the light speed. Thus, this point is one the main points in choosing the proper simulator. To simulate the speed of the optical lights, the amount of the traffic in the network simulator that generated in one second should be equal to the amount of traffic passes through the fiber optic in one second. Based on that, the network simulator should be able to generate the required amount of traffic.

One of the differences between the simulators is the modulebased structure (Wang et al., 2007). The NCTUns supports normal applications and general network applications, such as ftp and some TCP/UDP traffic generator programs to generate the traffic flow on the NCTUns, which can generate an enough amount of traffic for the OBS networks. On the other hand, other simulators use a mathematical modeling program or a specified traffic generator that generates the traffic flow by mathematical computation to simulate the traffic flow, which cannot meet the needed amount of real time traffic on the OBS network.

One other crucial issue in the module-based structure of the simulator that can help to get accurate results in the OBS network simulation is the control channel's simulation. Most of the current simulators simulate only the data channels, and they simply assume that no drop occurs in the control channels.

On the contrary, the actual scenario of the simulation can be identified using the control channels. In the actual scenario, if the control packet does not arrive at the core node, or it arrives late, the core node does not reserve the resources for the data channel bursts, causing it to drop the data burst. Thus, the simulation process must be the same process in the actual scenario.

The control channel's simulation is one of the advantages of NCTUns that is not available in the rest of the simulators. The control channel's simulation can easily be simulated in the NCTUns. Moreover, the control packet's parameters can be controlled, and the parameters can be displayed.

Furthermore, the control packets plots that show the actual states of the packets can be drawn in NCTUns. Table 1 shows the comparison among the simulators in case of Module base structure.

\subsection{Simulating Methodology}

The real simulating methodology, in which the simulator simulates the actual process as it is in the real experimentation, is another effective parameter in the simulator rustles. In the real network's experimentation, the packet is generated by the socket which puts the packet into the kernel.
Table 1 The comparison among the simulators in case of Module base structure

\begin{tabular}{|c|c|c|c|}
\hline $\begin{array}{c}\text { OBS } \\
\text { Simulators }\end{array}$ & $\begin{array}{c}\text { Traffic } \\
\text { Generator }\end{array}$ & $\begin{array}{c}\text { Control } \\
\text { Channel } \\
\text { Simulation }\end{array}$ & $\begin{array}{c}\text { Contr } \\
\text { ol packets } \\
\text { plots }\end{array}$ \\
\hline NCTUns & $\begin{array}{c}\text { Support } \\
\text { real network } \\
\text { applications }\end{array}$ & $\begin{array}{c}\text { Availab } \\
\text { le }\end{array}$ & $\begin{array}{c}\text { Avail } \\
\text { able }\end{array}$ \\
\hline OPNET & $\begin{array}{c}\text { Use a } \\
\text { mathematical } \\
\text { model }\end{array}$ & $\begin{array}{c}\text { Not } \\
\text { available }\end{array}$ & $\begin{array}{c}\text { Not } \\
\text { available }\end{array}$ \\
\hline OBS-ns & $\begin{array}{c}\text { Use a } \\
\text { mathematical } \\
\text { model }\end{array}$ & $\begin{array}{c}\text { Not } \\
\text { available }\end{array}$ & $\begin{array}{r}\text { Not } \\
\text { available }\end{array}$ \\
\hline OBSsimula \\
tor & $\begin{array}{c}\text { Use a } \\
\text { mathematical } \\
\text { model }\end{array}$ & $\begin{array}{c}\text { Not } \\
\text { available }\end{array}$ & $\begin{array}{c}\text { Not } \\
\text { available }\end{array}$ \\
\hline ADOBS & $\begin{array}{c}\text { Use a } \\
\text { mathematical } \\
\text { model }\end{array}$ & $\begin{array}{c}\text { Not } \\
\text { available }\end{array}$ & $\begin{array}{c}\text { Not } \\
\text { available }\end{array}$ \\
\hline JAVOBS & $\begin{array}{c}\text { Use a } \\
\text { mathematical } \\
\text { model }\end{array}$ & $\begin{array}{c}\text { Not } \\
\text { available }\end{array}$ & $\begin{array}{c}\text { Not } \\
\text { available }\end{array}$ \\
\hline
\end{tabular}

First, the packet is processed by the TCP/IP stack; which is then put by the kernel into the driver of the network interface card, which later pushes it into the network. In the NCTUns, the simulation process is the same as the operation in the real experimentation. NCTUns uses the OSI layer-3 and layer-4 protocol stacks of the operating system to simulate the TCP/IP protocol. In NCTUns, all simulated hosts, routers, and layer-3 devices use the same kernel TCP/IP stacks (as they are in the same computer) (Wang S.Y., 2009). OPNET modeler product uses a simplified finite state machine model to model the complex TCP protocol processing, whereas there is no dynamic receiver's advertised window for the TCP in the OBS-ns. Table 2 shows the comparison of the simulators in terms of simulation methodology and compilation.

\subsection{Compilation}

In the network simulator, the compilation process affects the performance of the other application programs, in which it must let another user-level application program run on top of the simulator. In OPNET and OBS-ns, the thread-supporting event scheduler, the application programs that generate the network traffic, the utility programs that configure, monitor, or gather statistics of a simulated network, the TCP/IP protocol implementation on hosts, the IP protocol implementation on routers, and links are compiled to form a single user-level program.

NCTUns provides UNIX POSIX API (i.e., the standard UNIX system called interface) with the real-life application programs to run normally and to let another user-level application program run on top of it, and as another operating system (Wang et al., 2003). OBSsimulator and ADOBS simulator use $\mathrm{C}++$ compilers to compile the programs. JAVOBS simulator is based on Java programming language. 
Table 2 The comparison of the simulators in term of simulation methodology and compilation

\begin{tabular}{|c|c|c|}
\hline $\begin{array}{c}\text { OBS } \\
\text { Network } \\
\text { Simulators }\end{array}$ & $\begin{array}{l}\text { Simulation } \\
\text { Methodology }\end{array}$ & $\begin{array}{l}\text { Simulation } \\
\text { Compilation }\end{array}$ \\
\hline NCTUns & $\begin{array}{l}\text { Uses the OSI layer-3 } \\
\text { and layer-4 protocol stacks } \\
\text { of the operating system }\end{array}$ & $\begin{array}{c}\text { provides } \\
\text { UNIX POSIX API }\end{array}$ \\
\hline OPNET & $\begin{array}{l}\text { Uses a simplified finite } \\
\text { state machine model }\end{array}$ & $\begin{array}{l}\text { All application } \\
\text { compiled to form } \\
\text { a single user-level } \\
\text { program }\end{array}$ \\
\hline OBS-ns & $\begin{array}{l}\text { No dynamic receiver's } \\
\text { advertised window for the } \\
\text { TCP }\end{array}$ & $\begin{array}{l}\text { All application } \\
\text { compiled to form } \\
\text { a single user-level } \\
\text { program }\end{array}$ \\
\hline $\begin{array}{l}\text { OBSsim } \\
\text { ulator }\end{array}$ & Use Script files & $\begin{array}{l}\text { Use C++ } \\
\text { compilers }\end{array}$ \\
\hline ADOBS & Predefined input file & $\begin{array}{l}\text { Use C++ } \\
\text { compilers }\end{array}$ \\
\hline $\begin{array}{l}\text { JAVOB } \\
\text { S }\end{array}$ & $\begin{array}{l}\text { Graphical, script or xml } \\
\text { input file }\end{array}$ & $\begin{array}{l}\text { Java based } \\
\text { compilation }\end{array}$ \\
\hline
\end{tabular}

\subsection{The Graphic User Interface (GUI)}

Graphic User Interface has a very important role in using the network simulator. The powerful Graphic User Interface provides the simulator user with the ability to easy set up and usage of a simulated network and application programs. NCTUns has a powerful Graphic User Interface which makes setup and usage of a simulated network and application programs very easy, similar to the ones used in the real world IP networks, as shown in Figure 1. In addition, the NCTUns provides a complete documentation either in the GUI user's manual or the protocol developer manual, as well as in the free website software ${ }^{1}$.

OPNET also has a GUI as shown in Figure 3 and it offers free professional training at the OPNET headquarters, lab manuals accompanying the best-selling 3rd party textbooks and an optional maintenance program which includes technical support, published documentation and best practice methodologies at a special academic price. The OPNET is commercial software, which can be found in the website ${ }^{2}$.

OBS-ns is free software with a GUI, as shown in Figure 2. However, it uses a command interface to set up and a simulated network which makes the usage of the simulator difficult. OBSns also provides documentation in how the user can use and setup the simulation model. The OBS-ns can be found in its website $^{3}$. For the rest of the simulators no GUI is provided.

\section{CONCLUSION}

In this paper, a comparison of sex simulators that provide the ability to simulate OBS network was introduced. The comparison among popular OBS simulators has been done based on the needs to simulate real time traffic in the OBS network and not to define which network simulator is the best as there are too many different parameters variations and different possible network scenarios to adequately determine this in a single section. The amount of the traffic and the simulation of the control channels were the main parameters of the choice of the network simulator.

The comparison results show that NCTUns will and can provide much better functions and performance as compared to other simulators in the simulation of real time traffic in OBS network, because of its several unique features, which are suitable for real time traffic in the OBS network. NCTUns can support normal applications and general network applications that can generate enough traffic for real time traffic. In addition, NCTUns uses the OSI layer-3 and layer-4 protocol stacks of the operating system to simulate the TCP/IP protocol. Moreover, it simulates the control channels as separate channels. Besides, NCTUns has a powerful and better Graphic User Interface which makes setup and usage of simulated network and application programs easy.

\section{REFERENCES}

[1] Tom Sheldon, and Big Sur Multimedia, (2001), Linktionar [Online]. Available: fttp://www.linktionary.com $/ \mathrm{m} /$ multimed ia.html

[2] Qiao, C., and Yoo, M. (1999). Optical Burst Switching (OBS) - a new paradigm for an optical Internet. Journal High Speed Networks, vol. 8, pp. 69-84.

[3] Wang, S.Y., Chou, C.L., Huang, C.H., Hwang, C.C., Yang, Z.M., Chiou, C.C., and Lin, C.C. (2003). The Design and Implementation of the NCTUns 1.0 Network Simulator. Computer Networks, Vol. 42, Issue 2, pp. 175-197. (EI)

[4] SimReal Inc., "NCTUns", http://nsl10.csie.nctu.edu.tw/, accessed at October, 2007.

[5] M. C. Yu, H. J. Tsai, C. Y. Huang, and S. Y. Wang, "Supporting Optical Network Simulations (OBS) on the NCTUns Network Simulator andEmulator", 2004.

[6] SimReal Inc., "NCTUns Support/Documentation web page", http://nsl10.csie.nctu.edu.tw/support/documentation/ mainframe.htm, accessed at October, 2007.

[7] Optical Internet Research Center, "OIRC OBS-ns Simulator", $\quad$ http://wine.icu.ac.kr/ obsns/index.php, accessed at October, 2007.

[8] "The Network Simulator - ns-2", http://www.isi.edu/nsnam/ns/, accessed at October, 2007.

[9] K. Fall and K. Varadhan, "The ns Manual (formerly ns Notes and Documentation)", http://www.isi.edu/nsnam/ ns/doc/index.html, accessed at October, 2007.

[10] "Tutorial for Optical Burst Switch Networks in NS2", http://wine.icu.ac.kr/ obsns/docs.php, accessed at October, 2007.

[11] Alexios Louridas, Kalliopi Panagiotidou and Nathan J. Gomes. (2002). Simulation of Optical Burst Switching Protocol and Physical Layers. London Communications Symposium 2002.

[12] McCanne, S., Floyd, S. (1996). ns-LBNL Network Simulator [Online]. Available: (http://wwwnrg.ee.lbl.gov/ns/).

\footnotetext{
${ }^{1}$ http://nsl10.csie.nctu.edu.tw

2 http://www.opnet.com

${ }^{3}$ http://wine.icu.ac.kr/ obsns /index.php
} 